\title{
Transcervical intrauterine bupivacaine for the management of postoperative pain following endometrial balloon ablation-a pre-trial toxicity study
}

\author{
Kalsang Bhatia • Oybek Rustimov • Manju Adhikary • \\ Simon Hill
}

Received: 8 August 2008 / Accepted: 15 October 2008 / Published online: 4 November 2008

(C) Springer-Verlag 2008

\begin{abstract}
The success of second-generation endometrial ablation performed as day case or outpatient procedure is often hampered by severe postoperative pain. We administered dilute bupivacaine solution into the uterine cavity soon after balloon ablation in ten consecutive women, looking at the safety profile of bupivacaine. Three hours postoperative serum bupivacaine levels were well below the toxic level in all women with no side effects reported.
\end{abstract}

Keywords Transcervical · Bupivacaine .

Postoperative pain relief Balloon endometrial ablation

\section{Introduction}

Postoperative analgesia is a key component of patient satisfaction following day case or ambulatory surgery. The success of second-generation endometrial ablation (EA) techniques as a day case or office procedure may be compromised by severe postoperative pain, which is known to occur from the release of prostaglandin from thermal destruction of the endometrium causing severe uterine cramping [1]. Recent studies have also shown that the onset of postoperative pain is often delayed irrespective of the type of anaesthesia, with onset typically about an hour after the procedure, peaking at 2-3 h often requiring opiates or antiemetics [2]. Although the need for postoperative pain relief following second-generation ablation is recognised,

\footnotetext{
K. Bhatia $(\triangle) \cdot$ O. Rustimov $\cdot$ M. Adhikary $\cdot$ S. Hill

Department of Obstetrics and Gynaecology,

Royal Blackburn Hospital,

Haslingden Road,

Blackburn BB2 3HH, UK

e-mail: Naviapo@aol.com
}

there are no published studies comparing different regimens for this purpose. There is, therefore, a need to optimise postoperative pain management for this group of women which will in turn help improve acceptability and utilisation of these procedures in the outpatient and day case settings.

It is now a standard practice to prescribe either $100 \mathrm{mg}$ diclofenac and/or $1 \mathrm{~g}$ paracetamol suppositories up to $1 \mathrm{~h}$ prior to the procedure or soon after the procedure. Majority of the time, when the procedure is performed under general anaesthesia, the postoperative pain management is at the discretion of the anaesthetist. It has been shown that up to $25 \%$ of women require additional opiate analgesia [2], and this figure is likely to be higher in endometrial balloon ablation with longer treatment times. The need for opiates in turn increases the potential for delayed transfer from recovery and discharge from hospital.

Local anaesthetic drugs act by causing a reversible conduction block along nerve fibres. They do not rely on the systemic circulation for transport to the site of action, but unwanted leak into the circulation is important in terminating their action and producing toxic side effects. The rate of systemic absorption of local anaesthetics is dependent upon the total dose and concentration of drug administered, route of administration, vascularity of the administration site and the presence or absence of epinephrine in the anaesthetic solution. It is, therefore, generally recommended that these compounds should not be used in inflamed or traumatised tissues. Systemic absorption of local anaesthetic compounds may affect the cardiovascular and central nervous systems, which, although minimal at the serum concentrations achieved with therapeutic doses, can be clinically significant with higher doses or greater systemic absorption. Toxic blood concentrations occur mainly from accidental intravascular injection, delayed degradation (hepatic and renal) and overdosage. Effects initially include 
a feeling of inebriation and light-headedness followed by sedation, circumoral paraesthesia and twitching; convulsions can occur in severe reactions. Cardiovascular effects include depression of cardiac conduction and excitability, leading to atrioventricular block, ventricular arrhythmias associated with peripheral vasodilatation, sometimes resulting in cardiac arrest. Recent clinical reports and animal research suggest that these cardiovascular changes are more likely to occur after unintended intravascular injection of bupivacaine [3].

Bupivacaine is preferred for postoperative analgesia due to its longer duration of action compared to other local analgesics. It takes up to $30 \mathrm{~min}$ for full effect with a halflife of $2.7 \mathrm{~h}$. The maximum therapeutic dose is $2 \mathrm{mg} / \mathrm{kg}$ with a toxic plasma level of $4 \mathrm{mg} / \mathrm{L}$. Dilute bupivacaine solution administered intraperitoneally is commonly used for postoperative pain relief after minimal access gynaecological and general surgery and their efficacy is evident in a number of randomised controlled trials [4-6]. Although no adverse effects have been reported, none of these studies measured serum bupivacaine levels or evaluated the side effect profile.

It is generally conceived that instillation of local anaesthetic solution into a uterine cavity treated with thermal ablation could accelerate its absorption with risk of toxicity, although there are no published studies to confirm this and these risks remain theoretical. There are, however, a number of studies reporting on the safety and efficacy of topical local anaesthetic solutions (lignocaine and bupivacaine) when used in traumatised and inflamed tissue, for example, severe burns and spinal surgery with serum levels below the toxic range [7, 8]. This has prompted us to plan a randomised controlled trial to test the postoperative analgesic efficacy of transuterine bupivacaine following second-generation endometrial ablation procedures. Due to the lack of published evidence on the safety of bupivacaine for this indication, we first conducted a small pre-trial toxicity study, the results of which are reported in this paper.

\section{Materials and methods}

This is a prospective pilot study conducted in a district general hospital in UK involving ten consecutive premenopausal women undergoing endometrial balloon ablation for dysfunctional uterine bleeding. All procedures were performed under general anaesthesia as day case. All women had normal cervical smear and endometrial biopsy and did not have any desire for future pregnancy. Only those women in whom the procedure was successfully completed were included. Women with cardiac disease, epilepsy or sensitivity to local anaesthetics were excluded from the study.
Ethical advice from the Local Research and Development Committee (LREC) was obtained and formal ethical approval was not needed for this study. Verbal and written information with consent forms as advised by the LREC was used.

The objective of the study was to determine the safety and feasibility of transcervical bupivacaine when instilled into the uterine cavity immediately after balloon ablation for the management of postoperative pain. Main outcome measures were serum bupivacaine levels ( $3 \mathrm{~h}$ postoperative) and the incidence of symptoms indicating clinical toxicity in the group.

All participants received postoperative analgesia as standard practice (intravenous or rectal diclofenac and/or paracetamol postoperatively) for the unit. The participants also received $50 \mathrm{~mL}$ of $0.25 \%$ bupivacaine $(125 \mathrm{mg}$, not exceeding $2 \mathrm{mg} / \mathrm{kg}$ ) which was instilled transcervically into the uterine cavity on completion of the procedure.

Consecutive women undergoing balloon ablation were offered the option of the new intervention in addition to standard treatment for postoperative pain relief. Written and informed consent was obtained. Demographic data including age, body mass index (BMI) and parity were collected. All procedures were performed under general anaesthetic, and balloon ablation was performed using Thermachoice ${ }^{\circledR}$ III (Gynecare, Ethicon, Somerville, NJ, USA). After the completion of the procedure, an appropriate-sized Foley catheter (depending on the dilatation of external cervical os) was inserted into the uterine cavity with $5 \mathrm{~mL}$ of water in the catheter balloon. Sufficient traction was placed upon the inflated balloon to occlude the internal os, and in Trendelenberg's position, $50 \mathrm{~mL}$ of $0.25 \%$ bupivacaine solution was injected through the catheter taking care to minimise any leakage. The time of bupivacaine administration was noted and a spigot was inserted into the catheter to allow retention of bupivacaine within the endometrial cavity for at least 15-20 min.

Whilst in the recovery room, usual monitoring for blood pressure and pulse rate was performed. Pain scores were recorded on a verbal scale of 0 to 4 , which is the standard practice for scoring postoperative pain in the recovery room for the East Lancashire Hospitals NHS trust ( 0 - none, $1-$ mild pain, 2-mild but moderate on movement, 3moderate but severe on movement and 4 - severe at rest). The catheter was removed approximately 15-20 min after the procedure before transfer to ward. Once on the ward, the co-investigator reviewed the participants enquiring into symptoms such as pain, palpitation, circumoral paraesthesia and twitching. A serum sample for bupivacaine levels was obtained $3 \mathrm{~h}$ after administration and any additional analgesia requirements were noted. Pain score at discharge was also recorded. All women were given analgesia to take home as usual (paracetamol and diclofenac).

Analysis of the serum samples were undertaken at the Medical Toxicology Laboratory, Guy's and St. Thomas' Hospital in London. 


\section{Results}

Ten women received bupivacaine for postoperative pain relief following Thermachoice ${ }^{\circledR}$ balloon ablation. The mean age was 43 years (range 33-49 years) and the mean BMI was $31.5 \mathrm{~kg} / \mathrm{m}^{2}$ (range $21-43 \mathrm{~kg} / \mathrm{m}^{2}$ ); all were parous. One blood sample was sent in the wrong bottle and, hence, only nine samples were analysed by the laboratory. None of the women reported symptoms indicative of bupivacaine toxicity. Serum bupivacaine levels ( $3 \mathrm{~h}$ postoperative) ranged between $<0.1$ and $1.3 \mathrm{mg} / \mathrm{L}$, being well below the toxic level $(4 \mathrm{mg} / \mathrm{L})$ in all women. Levels were negligible $(<0.1 \mathrm{mg} / \mathrm{L})$ in four women (see Table 1$)$.

Table 1 also shows the pain scores and postoperative analgesia received by the participants. None of the women received analgesia preoperatively: nine out of ten women received diclofenac and paracetamol soon after the procedure as part of standard practice for the anaesthetist concerned. Two out of ten women (both had uterine curettage to thin the endometrium prior to EA) also needed parenteral morphine. Both these women stayed for overnight observation since the procedures were performed in the late afternoons. The mean pain score for the group (scale of 0-4) was 1.1 (range 0-3) in the recovery room and 0.7 (range $0-1$ ) at discharge.

\section{Discussion}

This pilot study shows that, in the dosages used, intrauterine bupivacaine given soon after endometrial balloon ablation appears to be safe and free of any clinical toxicity, although this needs to be confirmed in a larger study.

A dilute solution of bupivacaine $(0.25 \%)$ was specifically used in this study to reduce the possibility of systemic absorption of bupivacaine from the heated endometrial surface. Toxic serum levels are associated mainly with accidental intravascular injection or overdosage; the risk of systemic absorption is likely to be minimal with topical administration of therapeutic doses. However, by occluding the cervical os by a Foley catheter as in this study, there is a potential for increased toxicity due to uncontrolled rise in intrauterine pressure (IUP) and, hence, caution needs to be exercised especially when giving larger doses. Hasham et al. conducted a small randomised controlled trial comparing IUP and fluid deficit in two groups of women undergoing endometrial laser ablation (ELA) with and without control of intrauterine pressures. They demonstrated that control of IUP during ELA was effective in preventing absorption of irrigating fluid [9].

Intraperitoneal bupivacaine has been used in dosages between 50 and $285 \mathrm{mg}$ for postoperative pain following laparoscopic procedures in general surgical and gynaecological practice and dosages of less than $100 \mathrm{mg}$ were not found to be effective [7, 8]. In this study, although a Foley catheter was used to occlude the cervical os, there is potential for leakage and, therefore, for the intervention to be effective, $125 \mathrm{mg}$ of bupivacaine was used without titrating for body weight, making sure the maximum therapeutic dose $(2 \mathrm{mg} / \mathrm{kg}$ ) was not crossed in smaller women.

The half-life of bupivacaine is $2.7 \mathrm{~h}$ and, hence, serum levels were taken at $3 \mathrm{~h}$ postoperatively. Serum bupivacaine after $3 \mathrm{~h}$ was well below the toxic level in all women - even double the measured levels were still well within the accepted safety limit. No side effects attributable to bupivacaine toxicity were reported in this small series, consistent with the serum levels.

It is difficult to ascertain whether the intervention was effective in relieving postoperative pain since almost all participants also received both diclofenac and paracetamol

Table 1 Serum bupivacaine levels, pain score and postoperative analgesia in study participants

\begin{tabular}{|c|c|c|c|c|c|c|}
\hline $\begin{array}{l}\text { Participant number } \\
\text { (study group) }\end{array}$ & $\begin{array}{l}\text { Serum bupivacaine } \\
(3 \mathrm{~h} \text { postoperative; } \mathrm{mg} / \mathrm{L})\end{array}$ & $\begin{array}{l}\text { Pain scores at recovery/ } \\
\text { discharge }^{\text {a }}\end{array}$ & Diclofenac & Paracetamol & $\begin{array}{l}\text { Other } \\
\text { analgesia }\end{array}$ & $\begin{array}{l}\text { Morphine } \\
\text { (total dose) }\end{array}$ \\
\hline 1 & $<0.1$ & $0 / 1$ & $100 \mathrm{mg}$ PR & $1 \mathrm{~g} \mathrm{PR}$ & Codeine $60 \mathrm{mg}$ & - \\
\hline $2^{\mathrm{b}}$ & 0.1 & $3 / 0$ & $100 \mathrm{mg}$ PR & $1 \mathrm{~g}$ iv & Codeine $60 \mathrm{mg}$ & $10 \mathrm{mg}$ \\
\hline 3 & Wrong sample & $0 / 0$ & $100 \mathrm{mg}$ PR & $1 \mathrm{~g} \mathrm{PR}$ & - & - \\
\hline $4^{b}$ & 0.5 & $2 / 0$ & $50 \mathrm{mg}$ iv & $1 \mathrm{~g}$ & Buscopan $10 \mathrm{mg}$ & $10 \mathrm{mg}$ \\
\hline 5 & $<0.1$ & $1 / 1$ & - & - & Codeine $60 \mathrm{mg}$ & - \\
\hline 6 & 0.1 & $1 / 1$ & $75 \mathrm{mg}$ iv & $1 \mathrm{~g} \mathrm{PR}$ & - & - \\
\hline 7 & 1.3 & $1 / 1$ & $50 \mathrm{mg}$ iv & $1 \mathrm{~g}$ iv & - & - \\
\hline 8 & $<0.1$ & $2 / 1$ & $100 \mathrm{mg}$ PR & $\begin{array}{l}1 \mathrm{~g} \mathrm{PR} \\
1 \mathrm{~g} \text { iv }\end{array}$ & Codeine $60 \mathrm{mg}$ & - \\
\hline 9 & $<0.1$ & $0 / 1$ & $75 \mathrm{mg}$ iv & $1 \mathrm{~g} \mathrm{PR}$ & Co-codamol $\times 2$ & - \\
\hline 10 & 0.5 & $1 / 1$ & $75 \mathrm{mg}$ iv & $1 \mathrm{~g} \mathrm{PR}$ & Codeine phos $60 \mathrm{mg}$ & - \\
\hline
\end{tabular}

${ }^{a}$ Pain score — verbal scale of 0-4 (standard use in recovery — East Lancashire Hospital NHS Trust; 0 —none, 1 -mild pain, 2-mild but moderate on movement, 3-moderate but severe on movement and 4-severe at rest)

${ }^{\mathrm{b}}$ Required overnight stay 
given immediate postoperatively as a prophylactic standard practice. Moreover, the study was not designed to assess this outcome measure. Two out of ten women in this study required parenteral opiates: a recent unpublished local audit reported an opiate requirement of $70 \%$ in women undergoing endometrial balloon ablation, reflecting the need to optimise postoperative care in this group of women.

For the intervention to be effective, it is important to reduce leakage from the cervix by obtaining a good seal, maintain Trendelenberg's position and slow injection into the catheter whilst allowing the uterus to relax when resistance is encountered from uterine contractility. The use of smaller volume of more concentrated bupivacaine solution may be another option. Bupivacaine with adrenaline helps slow systemic absorption to allow more sustained local anaesthetic effect and reduce toxicity and, hence, may be more appropriate for this purpose.

\section{Conclusion}

Intrauterine instillation of dilute bupivacaine solution (125 mg) soon after endometrial balloon ablation appears to be safe and feasible, although we appreciate that measurement of IUP whilst the Foley catheter was in situ would have provided additional valuable information. Our proposed randomised controlled trial could demonstrate a useful method of providing postoperative analgesia for endometrial ablation procedures.

Acknowledgement We would like to thank the Biochemistry Department at Royal Blackburn Hospital for supporting the study and arranging transportation of the blood samples and the Medical Toxicology Laboratory, Guy's and St. Thomas' Hospital in London for analysing the samples. No other additional funding was needed for this study.

Disclosure of interest None declared.

\section{References}

1. Lethaby A, Hickey M, Garry R (2005) Endometrial destruction techniques for heavy menstrual bleeding. Cochrane Database Syst Rev(4):CD001501

2. Wallage S, Cooper KG, Graham WJ, Parkin DE (2003) A randomised trial comparing local versus general anaesthesia for microwave endometrial ablation. BJOG 110:799-807

3. Mather LE, Chang DH (2001) Cardiotoxicity with modern local anaesthetics: is there a safer choice? Drugs 61(3):333-342

4. Moiniche S, Jorgensen H, Wetterslev J, Dahl JB (2000) Local anesthetic infiltration for postoperative pain after laparoscopy: a qualitative and quantitative systematic review of intraperitonieal, portsite infiltration and mesosalpinx block. Anesth Analg 90:899-912

5. Sripada S, Roy S, Mathur M et al (2006) A prospective doubleblind randomised controlled trial of introperative pelvic instillation with bupivacaine for management of pain following laparoscopy and dye. BJOG 113:835-838

6. Malhotra N, Chanana C, Roy KK, Kumar S, Riwari V, Sharma JB (2007) To compare the efficacy of two doses of intraperitoneal bupivacaine for pain relief after operative laparoscopy in gynecology. Arch Gynecol Obstet 276(4):323-326

7. Jellish WS, Gamelli RL, Furry PA, McGill VL, Fluder EM (1999) Effect of topical local anesthetic application to skin harvest sites for pain management in burn patients undergoing skin-grafting procedures. Ann Surg 229(1):115-120 (Jan)

8. Singh K, Samartzis D, Strom J, Manning D, Campbell-Hupp M, Wetzel FT, Gupta P, Phillips FM (2005) A prospective, randomized, double-blind study evaluating the efficacy of postoperative continuous local anesthetic infusion at the iliac crest bone graft site after spinal arthrodesis. Spine 30(22):2477-2483 (Nov 15)

9. Hasham F, Garry R, Kokri MS, Mooney P (1992) Fluid absorption during laser ablation of the endometrium in the treatment of menorrhagia. Br J Anaesth 68(2):151-154 (Feb) 